\title{
Lung Ultrasound (LUS) in COVID-19 Pneumonia: Usefulness in Two Atypical Cases
}

\author{
Vincenza Giugno, Giulia Di Marzio, Andrea Domanico, Esterita Accogli \\ Ultrasound Centre, Maggiore Hospital, Bologna, Italy
}

Received: 12/06/2020

Accepted: 15/06/2020

Published: $26 / 06 / 2020$

How to cite this article: Giugno V, Di Marzio G, Domanico A, Accogli E. Lung ultrasound (LUS) in COVID-19 pneumonia: usefulness in two atypical cases. EJCRIM 2020;7: doi:10.12890/2020_001800.

Conflicts of Interests: The Authors declare that there are no competing interests.

This article is licensed under a Commons Attribution Non-Commercial 4.0 License

\section{ABSTRACT}

Clinical experience and scientific articles have shown that patients infected with severe acute respiratory syndrome coronavirus 2 (SARSCoV-2) can be paucisymptomatic or asymptomatic at the time of diagnosis. In this paper, we will discuss two paucisymptomatic patients with blood tests suggestive for SARS-CoV-2 infection but with repeated negative nasopharyngeal swabs and without typical features of COVID-19 pneumonia on chest high-resolution computed tomography. In these cases, lung ultrasound helped to raise clinical suspicion of COVID-19 pneumonia and facilitate diagnosis.

\section{LEARNING POINTS}

- During the current COVID-19 pandemic, lung ultrasound (LUS) is being used extensively to evaluate and monitor lung damage in infected patients.

- Several patients have been described with negative PCR swabs who tested positive for SARS-CoV-2 in bronchoalveolar lavage fluid.

- Typical signs of interstitial pneumonia on LUS strongly indicate COVID-19 pneumonia, thus suggesting further investigation and invasive tests to confirm the diagnosis.

\section{KEYWORDS}

Lung ultrasound (LUS), coronavirus disease 2019 (COVID-19), high-resolution computed tomography (HRCT), nasopharyngeal swab (NS), pneumonia

\section{INTRODUCTION}

At the end of December 2019, an outbreak of pneumonia in Wuhan caused by severe acute respiratory syndrome coronavirus 2 (SARSCoV-2) resulted in a global pandemic [1]. The disease is not yet fully understood, but a huge amount of research on clinical symptoms and diagnostic investigations has been published. The main symptoms and signs of infection are fever, cough and dyspnoea, although approximately $80 \%$ of patients are paucisymptomatic or asymptomatic at the time of diagnosis.

Chest HRCT is the reference imaging tool for the diagnosis of COVID-19 lung lesions in patients with a positive nasopharyngeal swab (NS) RT-PCR test. More recent studies have documented patients with three or more negative swabs yet presenting with the typical clinical picture and chest CT features of COVID-19 pneumonia ${ }^{[2]}$. Lung ultrasound (LUS) has been used in patients with SARS-CoV-2 pneumonia and has proven useful in numerous clinical settings in association with chest CT for the early diagnosis of COVID-19 lung disease and, especially, for monitoring patients with SARS-CoV-2 pneumonia. Pleuroparenchymal lesions typical of COVID-19 pneumonia have been well documented on LUS ${ }^{[3]}$. Consequently, we wonder if LUS could also be useful in diagnosing early COVID-19 pneumonia even in paucisymptomatic patients (fever $\leq 37.5^{\circ} \mathrm{C}$, asthenia) who have laboratory tests suggestive for the disease (high interleukin- 6 and procalcitonin, neutropenia, and low eosinophils and lymphocytes) but persistently negative NS. 


\section{CASE DESCRIPTION}

We describe two patients attending our ultrasound centre for abdominal ultrasound for suspected haematological disease. Both patients, an 84-year-old man with persistent fever and a 76-year-old woman with fever and asthenia, had already undergone chest HRCT and NS. In neither patient did HRCT show any lung lesions suggestive of COVID-19 pneumonia, while the NS, repeated twice in the first case and three times in the second, was persistently negative. The abdominal ultrasound was normal. Given the clinical picture, ultrasonography was then extended to the lungs.

LUS was performed using a 2-5 MHz convex probe and 7.5-12 MHz linear probe. Each inter-costal space of the upper and lower parts of the anterior, lateral and posterior regions of the left and right chest wall (12 areas) was examined. In both patients, LUS showed findings suggestive for COVID-19 interstitial pneumonia. In these patients, a wide range of ultrasonographic patterns was seen, such as thickening and irregularity of the pleural line, variation of B line patterns including their number and distribution (focal and multifocal), and small parenchymal consolidations. These features are explained by the location of lung damage, which typically starts in the periphery of the lung and then extends centripetally. Figs. 1 and 2 show ultrasound images for the male patient and the corresponding chest HRCT findings, demonstrating irregularity, thickening and discontinuity of the pleural line (Fig. 1a) associated, in some areas, with B lines (Fig. 1b) and subpleural consolidations of different sizes in several areas (Fig. 2a,b).

Figs. 3 and 4 show lung ultrasound images for the female patient and corresponding chest CT images demonstrating irregularity and discontinuity of the pleural line associated with a small sub-pleural consolidation (Fig. 3a and Fig. 4a,b).

As the ultrasound results raised strong suspicion of COVID-19 interstitial pneumonia in both patients, bronchoalveolar lavage fluid (BAL) was obtained and tested positive for SARS-CoV-2 in each case.
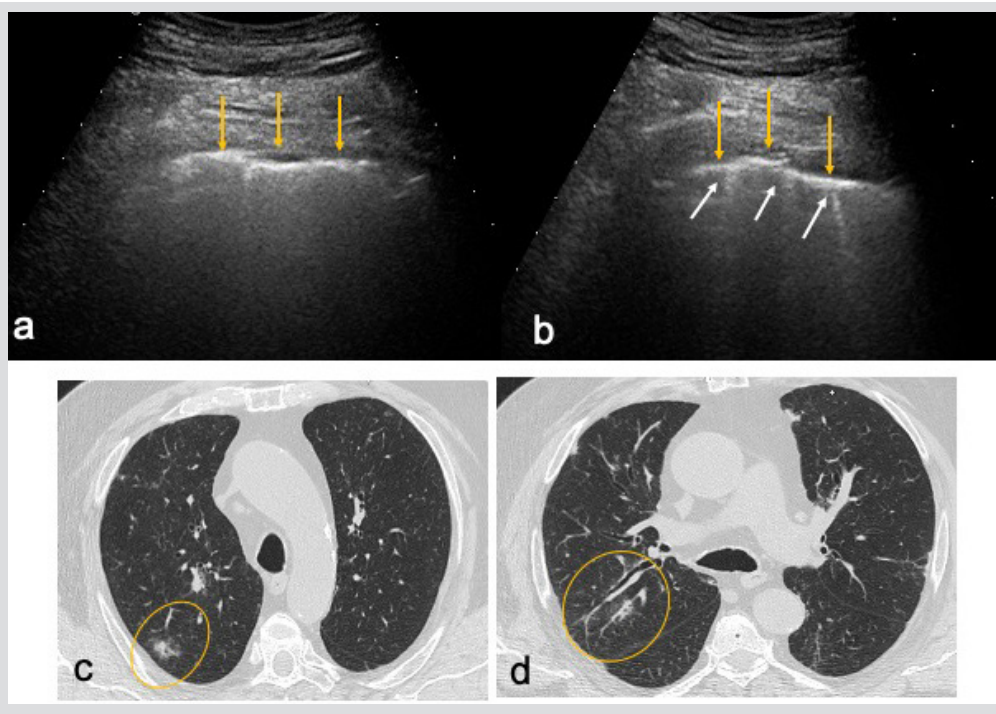

Figure 1. Right lung of patient 1. Lung ultrasound (LUS): (a) irregular, thickened and discontinued pleural line (yellow arrows); (b) irregular, thickened pleural line with three $B$ lines (white arrows). High-resolution computed tomography (HRCT): (c, d) areas corresponding to LUS features (circles)
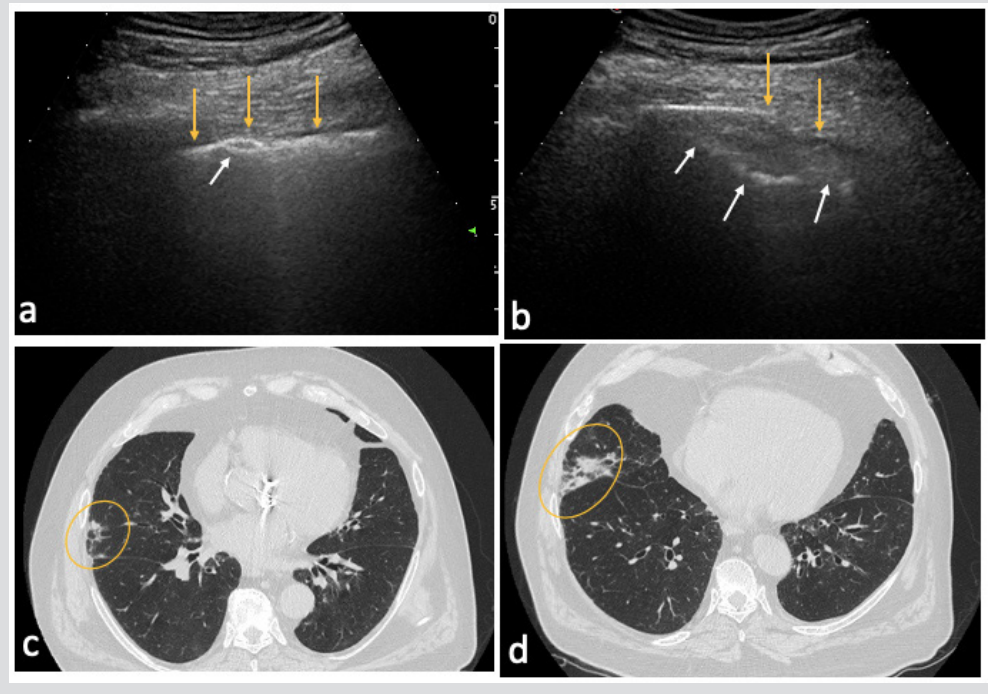

Figure 2. Right lung of patient 1. Lung ultrasound (LUS): (a) irregular, thickened and discontinued pleural line (yellow arrows) with small sub-pleural consolidation (white arrow); (b) discontinued pleural line (yellow arrows) with large sub-pleural consolidation (white arrows). High-resolution computed tomography (HRCT): (c, d) areas corresponding to LUS features (circles) 

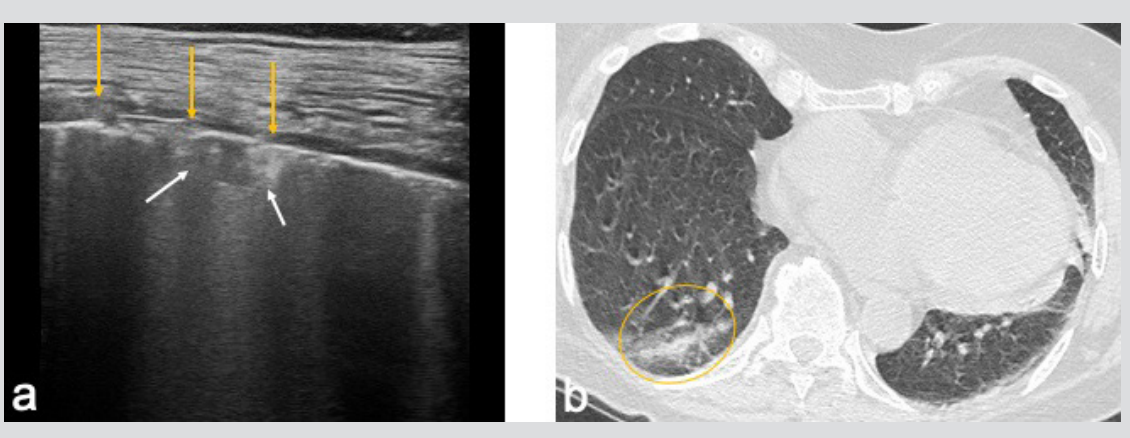

Figure 3. Right lung of patient 2. (a) Lung ultrasound (LUS): discontinued pleural line (yellow arrows) with small sub-pleural consolidation (white arrows); (b) High-resolution computed tomography (HRCT): areas corresponding to LUS features (circle)

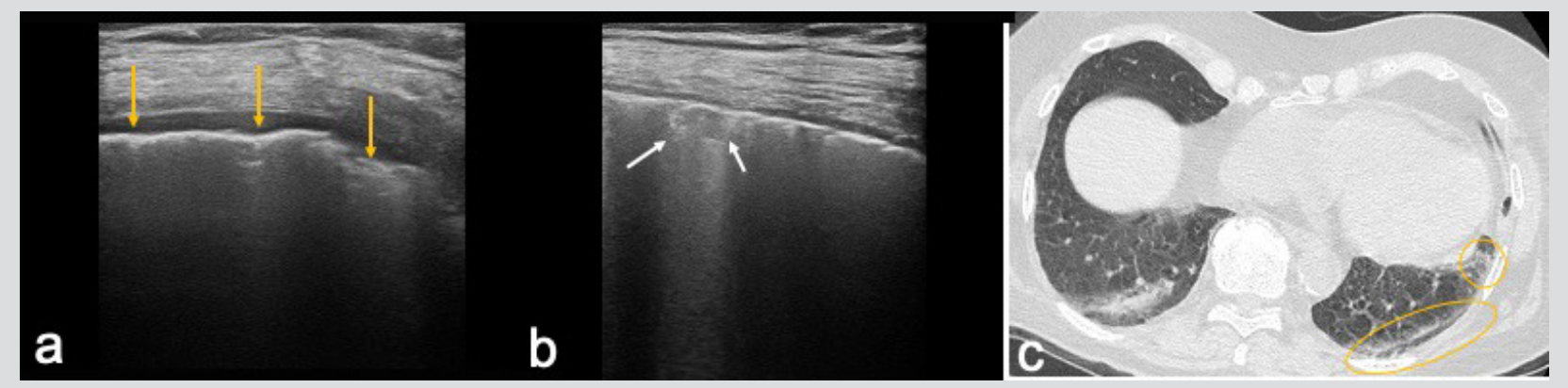

Figure 4. Left lung of patient 2. Lung ultrasound (LUS): (a) irregular and discontinued pleural line (yellow arrows); (b) small sub-pleural consolidation (white arrows). High-resolution computed tomography (HRCT): (c) the areas corresponding to LUS features (circles)

\section{DISCUSSION}

SARS-CoV-2 is a novel human pathogenic virus. In-depth research on its natural history and time course of infection is needed. Extensive study of its pathogenetic mechanism, which is related to cytokine release syndrome (CRS), has provided important information. While some patients develop severe disease, mainly pneumonia and respiratory failure leading to acute respiratory distress syndrome as a result of CRS and diffuse alveolar damage, some infected individuals remain completely asymptomatic and others show only mild and non-specific symptoms.

In the two cases described, chest HRCT did not show any lung lesions indicating SARS-CoV-2 infection, but LUS findings were very suggestive for interstitial COVID-19 pneumonia. According to the literature, SARS-CoV-2 infection has different pathogenetic mechanisms and time courses, leading to extremely variable clinical manifestations as regards symptoms, clinical history and lung parenchyma damage. Furthermore, we know that persistently negative NS is explained by low viral load ${ }^{[4]}$ but that viral particles have been isolated in $B A L^{[5]}$. In the two described cases, the NS was persistently negative and only after ultrasound revealed atypical features of lung damage, did both patients undergo BAL, which led to the diagnosis of COVID-19 pneumonia.

Finally, if the LUS findings in our patients were the result of early and peripheral atypical lung damage, then LUS could be used in asymptomatic or paucisymptomatic patients to support clinical suspicion of SARS-CoV-2 lung damage and suggest invasive diagnostic investigations to identify the virus (BAL).

\section{CONCLUSION}

In recent years, LUS has become an essential tool for clinicians. Initially developed for emergency and critical care settings, it is now used in internal medicine wards. In the current COVID-19 pandemic, it is being extensively employed to evaluate and monitor lung damage in patients, resulting in significant improvements in the management of patients.

As the clinical manifestations of SARS-CoV-2 infection can vary and lung parenchymal damage can be incremental and progressive, but also late and possibly atypical, we suggest that LUS could be used to detect typical signs of lung disease in paucisymptomatic patients and lower the threshold for conducting invasive investigations (BAL). 


\section{REFERENCES}

1. Zhu N, Zhang D, Wang W, Li X, Yang B, Song J, et al. A novel coronavirus from patients with pneumonia in China, 2019. N Engl J Med 2020;382:727-733.

2. Li D, Wang D, Dong J, Wang N, Huang H, Xu H, et al. False-negative results of real-time reverse-transcriptase polymerase chain reaction for severe acute respiratory syndrome coronavirus 2: role of deep-learning-based CT diagnosis and insights from two cases. Korean J Radiol 2020;21:505-508.

3. Peng QY, Wang XT, Zhang LN. Findings of lung ultrasonography of novel corona virus pneumonia during the 2019-2020 epidemic. Intensive Care Med 2020;46(5):849-850.[

4. Liu Y, Liao W, Wan L, Xiang T, Zhang W. Correlation between relative nasopharyngeal virus RNA load and lymphocyte count disease severity in patients with COVID-19. Viral Immunol 2020 Apr 10. doi: 10.1089/vim.2020.0062 [Epub ahead of print].

5. Xiong Y, Liu Y, Cao L, Wang D, Guo M, Jiang A, et al. Transcriptomic characteristics of bronchoalveolar lavage fluid and peripheral blood mononuclear cells in COVID-19 patients. Emerg Microbes Infect 2020;9:761-770. 